\title{
Commentary: Another important tool in the toolbox: The descending branch of the lateral circumflex femoral artery in coronary artery bypass grafting
}

\author{
Juan A. Abreu, MD, and Tom C. Nguyen, MD
}

\footnotetext{
From the Department of Cardiothoracic and Vascular Surgery, University of Texas Health Science Center Houston, McGovern Medical School, Houston, Tex.

Disclosures: Dr Nguyen reports Edwards LifeSciences (consultant); Abbott (consultant); and LivaNova (consultant). Dr Abreu has nothing to disclose with regard to commercial support.

Received for publication Sept 19, 2019; revisions received Sept 19, 2019; accepted for publication Sept 20, 2019; available ahead of print Oct 1, 2019.

Address for reprints: Tom C. Nguyen, MD, Department of Cardiothoracic and Vascular Surgery, University of Texas Health Science Center Houston, McGovern Medical School, 6400 Fannin St, Suite 2850, Houston, TX 77030 (E-mail: tom.c.nguyen@ gmail.com).

J Thorac Cardiovasc Surg 2021;161:1273-4

$0022-5223 / \$ 36.00$

Copyright (C) 2019 by The American Association for Thoracic Surgery

https://doi.org/10.1016/j.jtcvs.2019.09.097
}

We commend Luo and colleagues ${ }^{1}$ for their successful use of another conduit choice when performing coronary artery bypass surgery. Coronary artery bypass grafting has been employed as one of the main treatment options for coronary artery disease since the first half of the 20th century, initially in the form of the historical Vineberg operation. ${ }^{2}$ Fast forward to approximately 80 years later, the main conduits that are applied today are essentially the internal thoracic artery (left and right), the reverse greater saphenous vein, the radial artery, and the right gastroepiploic artery.

In this issue of the Journal, Luo and colleagues ${ }^{1}$ report their experience using the descending branch of the lateral circumflex femoral artery (DLCFA) in 36 consecutive patients in a single institution with a coronary target stenosis of at least $70 \%$. The practicality of this graft has been previously reported, especially as an option in reoperative coronary artery bypass grafting as reported by Tsuchida and colleagues ${ }^{4}$ in the Japanese literature.

Luo and colleagues ${ }^{1}$ enrolled 44 patients younger than 60 years of age over a 2-year period after applying carefully selected criteria during the preoperative screening phase using electrocardiogram-gated computed tomography angiography imaging. Some of the conditions included normal anatomical position of the vessel, diameter more than $1.5 \mathrm{~mm}$, and length of at least $10 \mathrm{~cm}$.

These characteristics were designated to decide whether the DLCFA could be harvested. Depending on the target location, the DLCFA was used either as a free graft or as a composite graft. The 1-year patency rate of all the conduits used during the study period were as follows: left internal mammary artery $95.4 \%$; radial artery $88.9 \%$; DLCFA $86.4 \%$; in situ right internal mammary artery (RIMA) $87.5 \%$; and free RIMA $75 \%$.

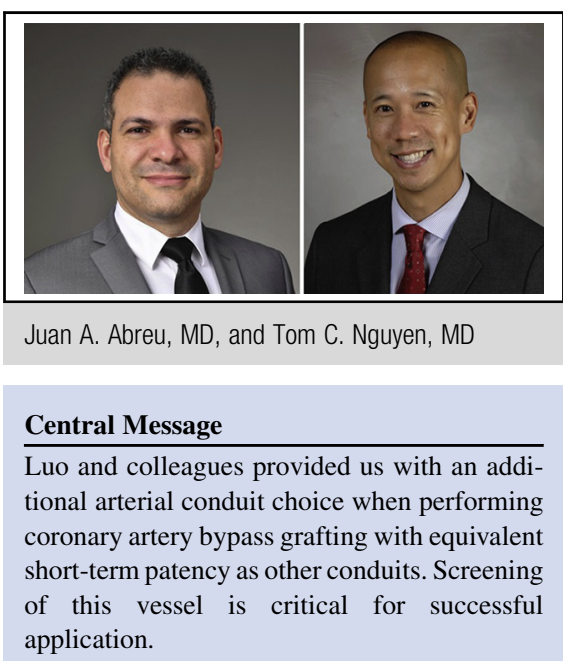

See Article page 1266.

A point that we would like to make is that these reported proportions are only short-term (1-year follow-up) and presenting a patency rate of $86.4 \%$, given this, one might suggest that is very close to the RIMA reported values. This finding is slightly lower that previously published data from the Italian group of Fabbrocini and collaborators, ${ }^{5}$ where in their experience, the 12-month patency was $97.6 \%$ and the 3 -year rate was $93.7 \%$, suggesting that the DLCFA comes second to the left internal mammary artery at least in the short term. This difference can be due to subtle variations either in the harvesting technique, preservation of the vessel, or prophylactic vasospasm protocol.

Interestingly, there was only a $50 \%$ cohort follow-up, suggesting that there might be patient compliance factors also equating into the final outcome. Taking this into consideration, we cannot generalize these findings, as they might not be an accurate representation of the reality.

In addition, the group encountered an $18 \%$ to $20 \%$ intraoperative abandonment rate of the conduit secondary to anatomical factors. Given this, we must think about this conduit not as a last resort option but more as an additional option in the armamentarium of coronary artery bypass surgery. This highlights the importance of an exceptional screening protocol very well described and applied by the authors, something that we cannot overlook.

In the end, Luo and his team did an excellent job detailing a specific preoperative screening process, and they 
described very well the harvesting technique of the DLCFA, demonstrating that it is a safe conduit to dissect and prepare, as there were no reported neuromuscular deficits in their report. Thanks to them, now we have another tool in the armamentarium of coronary artery bypass grafting surgery.

\section{References}

1. Luo XJ, Wang W, Wang YT, Yang Y, Li H, Wang X, et al. Application of the descending branch of the lateral circumflex femoral artery in coronary artery bypass grafting. J Thorac Cardiovasc Surg. 2021;161:1266-71.
2. Vineberg AM. Development of an anastomosis between the coronary vessels and a transplanted internal mammary artery. Can Med Assoc J. 1946;55:117-9.

3. Martínez-González B, Reyes-Hernández CG, Quiroga-Garza A, RodríguezRodríguez VE, Esparza-Hernández CN, Elizondo-Omaña RE, et al. Conduits used in coronary artery bypass grafting: a review of morphological studies. Ann Thorac Cardiovasc Surg. 2017;23:55-65.

4. Tsuchida T, Tatsumi T, Kondoh K, Sawada Y, Sasaki S. Descending branch of lateral femoral circumflex artery as a new alternative free graft in re-do CABG. Ann Thorac Cardiovasc Surg. 1999;5:130-2.

5. Fabbrocini M, Fattouch K, Camporini G, DeMicheli G, Bertucci C, Cioffi $\mathrm{P}$, et al. The descending branch of lateral femoral circumflex artery in arterial CABG: early and midterm results. Ann Thorac Surg. 2003;75: 1836-41. 